\title{
A Case of MRI Negative Metronidazole-Induced Encephalopathy and Recovery of Electroencephalography at Follow-Up
}

\author{
Halil Onder
}

\begin{abstract}
Metronidazole-induced encephalopathy (MIE) is an uncommon disease, presenting potentially with reversible acute developing encephalopathy due to the use of metronidazole. A 68-year-old female presented with a history of 1-week progressive confusion under metronidazole treatment. After discontinuation of metronidazole, her consciousness nearly totally recovered and normalization of electroencephalography (EEG) findings was ensured. On the basis of this patient, the diagnosis of MIE will be emphasized even in circumstances of negative magnetic resonance imaging (MRI) results. Dramatic recovery of EEG findings after treatment (solely discontinuation of metronidazole) will be demonstrated.
\end{abstract}

Keywords: Metronidazole; Encephalopathy; Electroencephalography; MRI

\section{Introduction}

Metronidazole is an antimicrobial agent which has been available for clinical use for more than 40 years and still plays an important role in the treatment of anaerobic infections as well as protozoan infections [1]. Metronidazole is accepted among blood-brain barrier penetrant antibiotics [2] and it causes various metronidazole-induced neurological side effects such as seizures, ataxia, encephalopathy and peripheral neuropathy [3, 4]. In addition, a recent report by Cantador et al demonstrated a patient with non-convulsive status epilepticus (NCSE) developing as a result of 10-day metronidazole therapy who had recovered after discontinuation of treatment [1]. On the other hand, metronidazole-induced encephalopathy (MIE) is a very rare complication especially occurring as a result of metronidazole usage exceeding $2 \mathrm{~g}$ /day dosages for prolonged periods [5]. Although cranial magnetic resonance imaging (MRI) findings

Manuscript accepted for publication July 20, 2016

Department of Neurology, Hacettepe University Hospital, Sihhiye, Ankara, Turkey. Email: halilnder@yahoo.com

doi: http://dx.doi.org/10.14740/jnr386w of this rare disease have been reported several times consisting of reports of small case series, electroencephalography (EEG) findings of MIE has not been previously mentioned in literature. Here, I present a patient diagnosed with MIE who totally recovered clinically and rapid normalization of EEG was supplied after discontinuation of metronidazole. Here, value of EEG, as an early indicator for diagnosis, will be discussed and also the necessity of MRI findings in the diagnostic algorithms will be questioned.

\section{Case Report}

A 68-year-old female who was diagnosed with cirrhosis associated with nonalcoholic steatohepatitis about 10 years ago was admitted to our center due to 1-week history of progressive confusion and ataxia. Umbilical hernia operation was performed 10 days before and 3 days after the operation, oral metronidazole $500 \mathrm{mg}$ three times daily was started due to diarrhea. At presentation, her vital signs were in normal ranges. On neurological examination, she was confused and disoriented in time and place, which were evaluated as encephalopathy. Not any abnormal focal or lateralized features were detected on neurological evaluations. Biochemical screening results (serum electrolytes, arterial ammonia, arterial blood gas, and complete blood count) were unremarkable except moderate hyponatremia $(125 \mathrm{mEq} / \mathrm{L})$ which was evaluated as chronic hyponatremia. Cranial MRI revealed bilateral pallidal hyperintensity on T1 sequences which were evaluated as imagining changes due to chronic cirrhosis (Fig. 1). Routine EEG recording showed diffuse, continuous theta-delta rhythm compatible with encephalopathy (Fig. 2). Although neuroimaging findings were unremarkable (except chronic effects of cirrhosis), the possible diagnosis of MIE was considered. Hence, metronidazole was discontinued and intravenous hydration therapy (saline) was started for hyponatremia. In the following days, her neurological examination gradually recovered and on the fourth day of treatment, she was completely oriented and cooperative. Simultaneous EEG revealed parietooccipital alpha rhythm (Fig. 3). However, laboratory investigations revealed continuation of hyponatremia which was evaluated as chronic hypervolemic hyponatremia $(126 \mathrm{mEq} / \mathrm{L})$. The only change was the discontinuation of metronidazole therapy in the interval period. Taken together, the diagnosis of MIE was made and she was discharged after achievement of a slight 


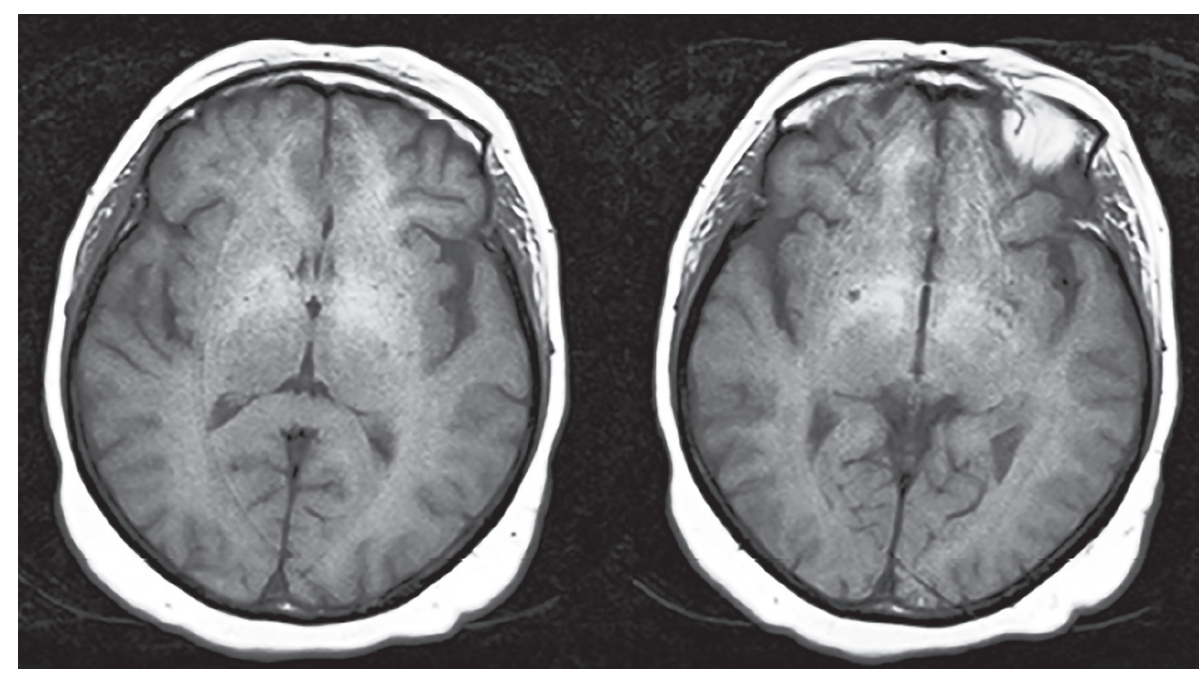

Figure 1. MRI showing bilateral globus pallidus T1 hyperintensities.

improvement in hyponatremia with fluid restriction therapy.

\section{Discussion}

Here, a rare patient with MIE was reported presenting with encephalopathy who totally recovered in a short time interval clinically and electroencephalographically recovery was shown after stopping metronidazole treatment. Interestingly, cranial MRI findings were unremarkable; nonetheless, dramatic recovery of both clinical and EEG findings after solely discontinuation of metronidazole enabled to make the diagnosis of MIE.

MIE is an uncommon and potentially reversible disease presenting with acute developing encephalopathy due to the use of metronidazole [6]. Although underlying mechanisms still remain to be clarified, there are some possible theories focused on. One of these theories was that metronidazole competes with nuclear thiamin leading to inhibition of its phosphorylation and the neurological complications occur via inhibition of intestinal thiamin absorption [7]. Ahmet et al, who described the first reversible MRI findings of MIE, explained the reversibility of the lesions via the mechanism of axonal swelling with increased water content rather than an underlying demyelination [3]. Also, imagining studies on patients with MIE frequently demonstrated both high diffusion-weighted imaging (DWI) and apparent diffusion coefficient (ADC) values in lesion sites which can be attributed to vasogenic edema, on the other hand, decreased ADC values were also found in a lower frequency of patient groups [8]. Affected areas reported include cerebellar dentate nucleus, midbrain, dorsal pons, medulla, and bilateral splenium of corpus callosum; however, the most commonly mentioned lesion sites among them are cerebellar dentate nuclei and inferior colliculus [3, 4]. On the other hand, EEG findings of a patient with MIE have not been reported before. Only, in a unique report, EEG findings of a patient with metronidazole-induced NCSE were demonstrated [1]. Hence, this report constitutes the first report demonstrating EEG findings of a patient with MIE in literature, hence presents very critical information emphasizing EEG as a crucial alternative tool in diagnosis of MIE in early phase.

Nonetheless, an important argument in this case may be the possible contributions of hyponatremia and chronic cir-

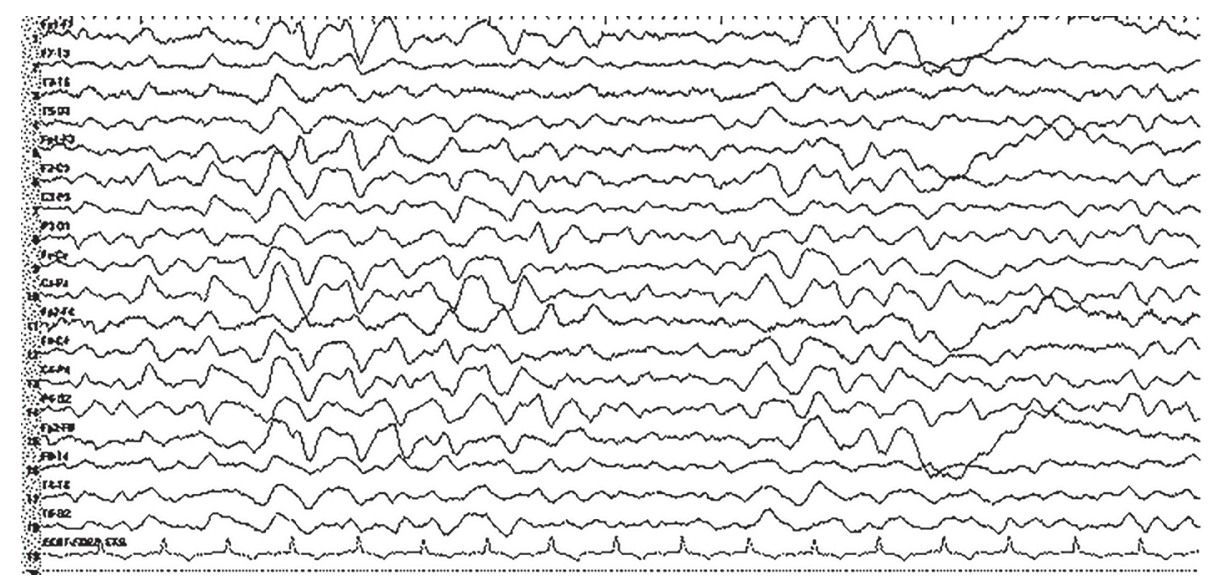

Figure 2. EEG at admission showed diffuse slowing in delta-theta rhythm. 


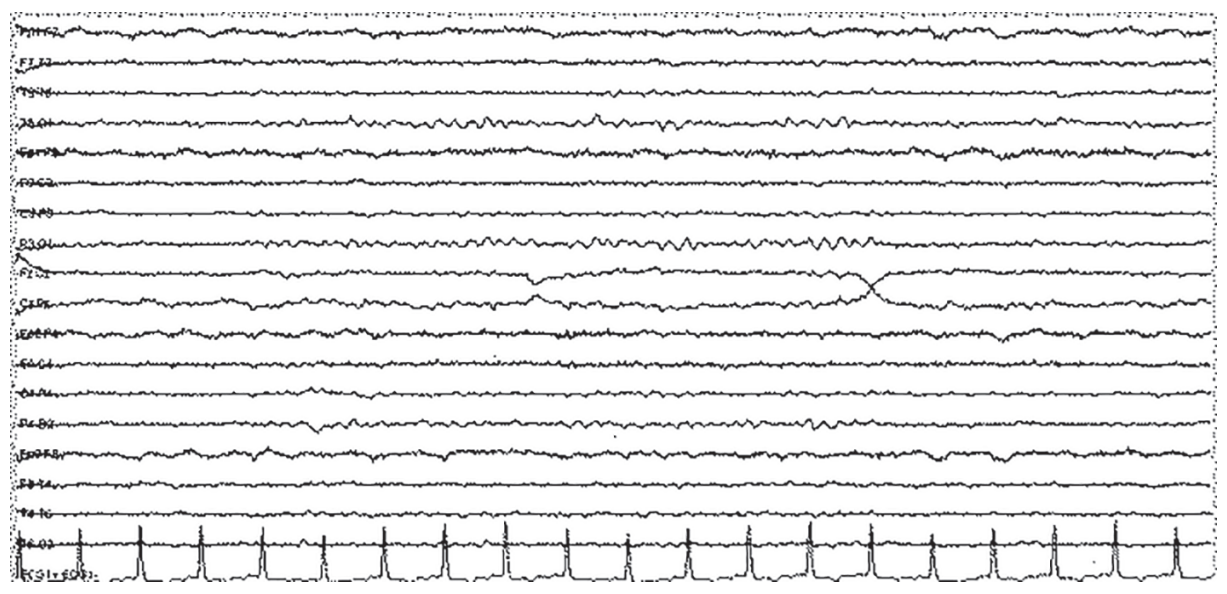

Figure 3. Follow-up EEG 4 days later from admission showed normal parietooccipital alpha rhythms.

rhosis in the manifestation of encephalopathy. Hyponatremia is one of the most common electrolyte abnormalities, affecting $2.5 \%$ of hospitalized patients [9]. Slowing of the posterior basic rhythm in hyponatremia has been reported at early phase of encephalopathy as well as more diffuse slowing in the delta range in subsequent periods [10]. Remarkably, it has been reported that EEG may remain abnormal for a while even after prompt correction of the hyponatremia [11]. Additively, EEG findings do correlate basically with alternation of serum sodium rather than absolute serum sodium levels [10]. In this case, laboratory investigations 1 week before symptom onset had revealed hyponatremia $(127 \mathrm{mEq} / \mathrm{L})$ and also at the time of totally clinical recovery (4 days after admission), hyponatremia $(126 \mathrm{mEq} / \mathrm{L})$ still continued. In light of these clinical data, it can be interpreted that there was not any correlation between serum sodium levels and patient's clinical course and EEG findings which pointed out sodium as an innocent agent in the clinical manifestations of the patient.

On the other hand, hepatic encephalopathy (HE) which is a syndrome observed in patients with cirrhosis or hepatic failure may constitute another differential diagnosis. The diagnosis requires impaired liver functions and exclusion of an alternative explanation for the symptoms. The pathophysiology underlying HE has been explained, to some extent, by the effect of neurotoxic substances occurring in the setting of cirrhosis [12]. Overt HE occurs in about $30-45 \%$ of patients with cirrhosis [12]. Serum ammonia levels can be useful in the diagnosis and it may be elevated in $90 \%$ of HE patients. EEG may not show any significant abnormalities in initial phase, but triphasic waves over frontal lobes can be seen in encephalopathy stages 1-3 as well as slow delta waves in most advanced stages [13]. Although serum ammonia level was normal in our case, a possible contributor effect of HE aggravated by hyponatremia still cannot be totally excluded. Nonetheless, EEG background rhythm was extremely slow which rapidly recovered after solely metronidazole cutoff, and serum sodium level was stabile during follow-up; moreover, there was not any factor suggestive to induce HE.

The patient had a history of metronidazole treatment for 7 days (total dosage: $10.5 \mathrm{~g}$ ) due to post-surgery diarrhea. EEG at admission confirmed the diagnosis and after discontinuation of metronidazole, nearly totally improvement was achieved clini- cally and the normalization of EEG was shown in the fourth day of follow-up. MRI did not show any findings that could be associated with MIE. Most brain lesions induced by metronidazole are reversible and resolve within several weeks after cutout of metronidazole [6]. Nonetheless, the longer duration of metronidazole usage with more cumulative doses is known to increase the risk of toxicity and worsen the prognosis $[6,7]$. The patient was admitted to our clinic on the initial stage of encephalopathy (seventh day of metronidazole usage) and I think that MRI lesions had not occurred due to the intervention at the early disease course. In literature, only a few reports have mentioned about MIE patients with unremarkable imaging findings $[14,15]$. One possibility may be that several patients with MIE might have been undiagnosed based on the unremarkable neuroimaging results performed in the early phase of the disease as in this case. Hence, the patient presented in this report may give crucial points regarding the knowledge of MIE. I suggest that clinicians should keep in mind metronidazole as a probable cause of encephalopathy in circumstances of a history of metronidazole usage, even in the cases of normal cranial MRI results. However, future studies with larger case series have to be conducted to clarify these arguments which may add crucial perspectives in the evaluation of these patients.

\section{Conflicts of Interest}

The author HO declares no potential conflicts of interest with respect to the research, authorship, and/or publication of this article.

\section{Funding}

HO did not receive any financial support for the research.

\section{Abbreviations}

DWI: diffusion-weighted imaging; ADC: apparent diffusion 
coefficient

\section{References}

1. Cantador AA, Meschia JF, Freeman WD, Tatum WO. Nonconvulsive status with metronidazole. Neurohospitalist. 2013;3(4):185-189.

2. Jokipii AM, Myllyla VV, Hokkanen E, Jokipii L. Penetration of the blood brain barrier by metronidazole and tinidazole. J Antimicrob Chemother. 1977;3(3):239-245.

3. Ahmed A, Loes DJ, Bressler EL. Reversible magnetic resonance imaging findings in metronidazole-induced encephalopathy. Neurology. 1995;45(3 Pt 1):588-589.

4. Woodruff BK, Wijdicks EF, Marshall WF. Reversible metronidazole-induced lesions of the cerebellar dentate nuclei. N Engl J Med. 2002;346(1):68-69.

5. Frytak S, Moertel CH, Childs DS. Neurologic toxicity associated with high-dose metronidazole therapy. Ann Intern Med. 1978;88(3):361-362.

6. Huang YT, Chen LA, Cheng SJ. Metronidazole-induced Encephalopathy: Case Report and Review Literature. Acta Neurol Taiwan. 2012;21(2):74-78.

7. Kapoor K, Chandra M, Nag D, Paliwal JK, Gupta RC, Saxena RC. Evaluation of metronidazole toxicity: a prospective study. Int J Clin Pharmacol Res. 1999;19(3):8388.
8. Kim E, Na DG, Kim EY, Kim JH, Son KR, Chang KH. MR imaging of metronidazole-induced encephalopathy: lesion distribution and diffusion-weighted imaging findings. AJNR Am J Neuroradiol. 2007;28(9):1652-1658.

9. Faigle R, Sutter R, Kaplan PW. Electroencephalography of encephalopathy in patients with endocrine and metabolic disorders. J Clin Neurophysiol. 2013;30(5):505-516.

10. Rebelo F, Conseiller C, Hazemann P. EEG study of a case of water intoxication. Electroencephalogr Clin Neurophysiol. 1971;30(3):254.

11. Reddy VR, Moorthy SS. Electroencephalographic and clinical correlation of hyponatremia induced during transurethral resection of the prostate. Ann Neurol. 2001;50(4):554-555.

12. Poordad FF. Review article: the burden of hepatic encephalopathy. Aliment Pharmacol Ther. 2007;25(Suppl 1):3-9.

13. Cash WJ, McConville P, McDermott E, McCormick PA, Callender ME, McDougall NI. Current concepts in the assessment and treatment of hepatic encephalopathy. QJM. 2010;103(1):9-16.

14. Boyce EG, Cookson ET, Bond WS. Persistent metronidazole-induced peripheral neuropathy. DICP. 1990;24(1):19-21.

15. Arik N, Cengiz N, Bilge A. Metronidazole-induced encephalopathy in a uremic patient: a case report. Nephron. 2001;89(1):108-109. 